\title{
INDICATIONS FOR THE REMOVAL OF TEETH IN CASES OF FOCAL INFECTION.
}

\author{
By Virgil Loeb, A. B., M. D., D.D.S., St. Louis, Mo.
}

(Read before the National Dental Association at Its Twentieth Annual Session, Louisville, Ky., July 25-28, 1916.)

I NVESTIGATIONS during the past few years have demonstrated without question the intimate relationship between focal infection around the teeth and certain systemic disorders. It is needless to enter into a discussion of this subject because of the great amount of valuable work which has already been done. My purpose is to discuss the indications which point toward the more or less radical removal of teeth where definite areas are found in the alveolar sockets from which toxines are being absorbed.

Prior to the common use of the X-ray, definite conclusions were indeed impossible as to the fate of many teeth. However, at the present time when no examination is complete without a roentgenograph of each tooth socket, it is much easier to determine which teeth should be saved, and which removed. It is of course the duty of every practitioner to conserve the teeth as far as possible, but not at the expense of the health of the patient. I do not wish to appear radical in my views, yet I feel that too often teeth are allowed to remain in the mouth which are detrimental in many ways.

The removal of teeth in this particular must depend on the following:

1. Relation of the infected area to the general condition of the patient. If the patient is absorbing a great amount of toxine from around a tooth and it is advisable to obtain immediate relief from this, the tooth should be removed whether or not it is amendable to treatment. In such cases the health of the patient may be seriously at stake and it may be imperative to relieve certain symptoms immediately. If a bacteriological examination shows a type of organism which is held to be responsible for the symptoms from which the patient suffers, immediate removal should be advised.

2. Relation of affected tooth to adjoining teeth. If an affected tooth is in such a condition that its retention is detrimental to adjoining teeth, this tooth should be removed, providing these adjoining teeth are of use to the patient. Often cases are found where the affected tooth can be benefited, tho not cured, and its retention is advised, even tho adjoining teeth may be affected by its presence. This is done in cases where the adjoining teeth are not of great value, or at least not of as great value as the one affected.

3. Condition of the alveolar socket. If the alveolus has been destroyed beyond the bifurcation of the roots of molar teeth, the teeth should in practically all cases be removed. Of course there are exceptions but these are rare. Often we find an isolated molar with only a slight amount of pus present, but the alveolar process absorbed much beyond the bifurcation. This tooth may be retained providing it is of value to the patient. In single rooted teeth where an insufficient amount of bony support is present, and where the infectious process is beyond control, immediate removal is indicated. Such teeth are often retained for cosmetic reasons, but every effort should be made to rid the mouth of pockets which do not yield to treatment. Too often the practitioner is guided by the wishes of 
his patients, in such cases, to their eventual detriment.

4. The relation of a tooth to mastication and the use to which such a tooth may be put in restoring masticatory surfaces. This is worthy of deep consideration for it is often necessary to determine whether an affected tooth is not less detrimental to a patient than the absence of a proper chewing surface. In other words we often find cases in which it is more necessary to construct a bridge of denture, using an affected tooth as an abutment, in order to give the patient sufficient surface to masticate the food properly, than to remove the seat of infection and leave the patient without a useful mouth. In such cases the amount of toxines absorbed may be less injurious than the swallowing of food improperly prepared for entrance into the stomach. In many mouths where pyorrhea has caused the loss of most of the teeth, there is often insufficient amount of alveolar process left to support a denture properly with. out the aid of a tooth around which a clasp may be swung. We must be ex. tremely guarded in our decision, before we endeavor to rid the mouth entirely of every seat of infection by removing teeth which may be the only. basis for the con. struction of proper dental appliances for mastication of food.

5. Exposed portions of roots which cannot be kept in a sanitary condition. Often a strongly imbedded root may be exposed, but. collects and retains food matter on account of the inability of the patient to cleanse the exposed surface. In these cases it is better to remove such teeth and to insert sanitary artificial ones than to allow the food matter to collect and decompose and thereby promote infection.

There are numerous conditions such as eroded apices, perforated roots, occluded canals, etc., which may call for the re. moval of the teeth. Often, however, such conditions may be present without caus- ing the patient any harm. In such cases these teeth may be allowed to remain in the mouth but whenever such a condition is accomplished by infection, the affected tooth should be removed, unless resection of the roots or other Iocal measures can be employed to relieve the condition permanently.

In the many conditions mentioned, which demand the removal of a tooth one fact must not be overlooked, and this is the possibility of local treatment. My statements have been made to apply to the general run of cases, and yet I do not wish to be understood as ignoring the possibility of relieving a specific case with treatment. Any one of the conditions mentioned may be amendable to treatment in isolated cases, but for the most part, the indications point toward the removal rather than the palliative plan.

I do not believe it is good practice to permit a patient to retain any condition which causes the absorption of toxines, and in case of doubt as to the efect of local treatment I believe we are always justified in being radical and removin? the offending tooth. This may seem contrary to what I have advised as to the retention of an affected tooth for purposes of increased efficiency for the patient. However, such retention is only advised when the infection is of a mild type and where the affected tooth is of value to the patient.

As already stated, roentgenographs must be obtained in order to determine the exact condition of the alveolar sockets. It is of the greatest advantage for study to arrange these in some convenient manner so that the normal relationship may be preserved. As this cannot be accomplished where pictures are mounted separately, they should be displayed collectively and in order, as seen in the slides which are exhibited.

The slides which I shall show represent cases of focal infection which illustrate the conditions I have previously mentioned. 